\title{
Generation and nomenclature of tessellations and double-layer grids
}

Valentin Gomez-Jauregui ${ }^{1}$, Cesar Otero $^{2}$, Ruben Arias $^{3}$, Cristina Manchado $^{4}$

\section{Abstract}

The aim of this work is to establish a systematic methodology for generating automatically different tessellations and double-layer grids (DLGs) following a defined and specific nomenclature proposed originally for such a task. This particular nomenclature defines the notation of mosaics and DLGs in a synthesized and unique manner, with the advantage that it shows how to generate and design them after the parameters expressed on their own names. As a result, by means of an algorithm and some computational codes, it is possible to recreate in 3D any of those grids directly from their own names.

Current nomenclature for tessellations is also analyzed, finding severe disadvantages, such as the excessive length of their notations or their non-uniqueness character. A new nomenclature is proposed in order to define and generate consistently and unequivocally n-uniform mosaics in a consistent manner with the current nomenclature used for the Archimedean (regular and semiregular) tessellations.

\section{Subject Heading}

Structural engineering, Architectural engineering, Geometry, Grid systems, Trusses, Space structures, Structural design.

\section{Keywords}

Double-layer grids, space structures, tessellations, mosaics, demiregular, n-uniform, nomenclature.

\footnotetext{
${ }^{1}$ Corresponding author: B.Eng, MSc Arch. Assistant lecturer, University of Cantabria, Spain. Tel. +34 620956 969, E-mail address: valen.gomez.jauregui@gmail.com. Dpt. Geographic Engineering and Graphical Expression Techniques. Avda./ Castros s/n. 39005 Universidad de Cantabria, Spain

${ }^{2}$ Dpt. Geographic Engineering and Graphical Expression Techniques. Avda./ Castros s/n. 39005 Universidad de Cantabria, Spain

${ }^{3}$ Dpt. Geographic Engineering and Graphical Expression Techniques. Avda./ Castros s/n. 39005 Universidad de Cantabria, Spain

${ }^{4}$ Dpt. Geographic Engineering and Graphical Expression Techniques. Avda./ Castros s/n. 39005 Universidad de Cantabria, Spain
} 


\section{Introduction}

\section{Introduction to double-layer grids}

Double-layer grids (DLG) may be defined as spatial systems containing two parallel networks of members forming the top and bottom chords in form of tessellations, whose nodes are linked by vertical and/or inclined web members (Malla and Serrette 1996).

Nowadays, there is a severe lack related to the configuration of DLG, specially concerning to the current nomenclature. The most usual and very basic grids are named by most of the authors (Eekhout 1989), (Makowski 1968; Makowski 1981), (Cuoco and American Society of Civil Engineers. Task Committee on Double-Layer Grids 1997), (Lan 1999), after the disposition of the bottom and top layer, taking some of these definitions: square-on-square, square-on-diagonal square, triangle-ontriangle, triangle-on-hexagon, etc. Sometimes, particles like "offset", "set orthogonally", "set diagonally", "hybrid chordal geometry" are added for contemplating more variants. Nevertheless, this classification is not specific enough to embrace all the possibilities proposed until now and all the others that are going to be presented in this paper. Along this work, some other names will be taken into account, according to some of the following illustrations from Makowski (1968), e.g., Space Deck (il. 69), Wachsmann (il. 71), Osaka (il. 72), Friedman (il. 73), Le Ricolais (il. 74), Hexagonal (il. 76.4), etc. Some others respond to designations used by Otero (1990), like Mero I and Unit IV. All of them will be referenced to along the text, so they will be illustrated at the end of the paper.

\section{Introduction to tessellations}

Regular tilings are those isomorph partitions of the Euclidean plane that accomplish strictly three conditions related to the polygons that conform them: 1 , all the polygons are regular; 2 , any vertex can be mapped to any vertex figure by symmetry operations which are transitive (i.e. vertices are congruent); and 3, all the polygons are equal, i.e. there is only a type of polygon. Thus, both vertex figures and faces are regular and congruent. There are only three mosaics meeting these conditions, composed by equilateral triangles, squares and regular hexagons. 
Semiregular tessellations, also called Archimedean, uniform, 1-uniform or homogenous, accomplish the first two conditions, but not the third one. Thus, they are polymorphic, that is, they are constituted by regular polygons of different number of edges concurring to any vertex and always respecting the same configuration or another analogous one obtained by means of reflections, rotations or translations. So, vertex figures are congruent but not regular, and faces are regular but not congruent. It is consistently proved that there are only eight exemplars of semiregular tilings (Critchlow 1969; Grünbaum and Shephard 1986). As an example, the mosaic 4,6,12 is formed in such a way that there is a square, a hexagon and a dodecagon concurring at every vertex.

Demiregular or $\mathbf{n}$-Uniform mosaics are, finally, those that only meet the first condition. In all their configurations, the arrangement of the vertices is not unique, but their regular polygons converge in several different ways (i.e, it is a tessellation with $\mathrm{n}$ transitivity classes). That is why they are also called No Uniform, $\mathrm{n}$-Uniform or $\mathrm{n}$-Isogonal (where $\mathrm{n}$ is greater than unity).

\section{Aims and intentions}

It is important to emphasize that in no way there is a pretension of recreating another algebraic representation, like Formex Algebra or Formian does. Both of them provide the tools to generate data for model structural systems in a parametric and concise manner or for obtaining their graphical visualization. Those tools don't seek to name and distinguish the different configurations following topological and geometrical rules, but constructing a whole algebraic basis including coordinate systems, symmetry operations (translations, reflections, rotations, etc.), boolean operations, projections, transformations to 3D surfaces on the space, etc.

The aim of this work is to establish a systematic nomenclature for defining the notation of tessellations and DLGs in a synthesized and unique manner, with the advantage that the proposed notation shows how to generate and design them after the parameters expressed on their own names. As a result, by means of an algorithm and some computational codes, it is possible to recreate in $3 D$ any of those grids directly from their own names. 


\section{Current nomenclature of tessellations}

\section{Cundy \& Rollett's notation}

Generally, in the mainstream literature, the notation suggested by Cundy and Rollett (1981) in the first edition of his book Mathematical Models, 1951, is often used for naming both the tessellations and polyhedra. The authors explain that this is a modification of the symbolism of Schläfli, which lists in order, clockwise and separated by dots (.), the number of sides of polygons surrounding each different vertex. For simplicity, when these numbers are repeated, a superscript indicates the number of repetitions of a given polygon; e.g. regular mosaic 6.6 .6 becomes $6^{3}$. Although in the original proposal Cundy and Rollett did not deal with the case of demiregular tiles, today, and most widely accepted, in the case of different configurations in different vertices, groups of polygons surrounding each vertex are listed separated by slashes (/). The authors also suggested adding, as a prefix, the letter ve and a point (V.) to the notation of any polyhedron or mosaic to name their corresponding duals.

For example, in the central image of Fig. 1, it is shown the confluence of three triangles ( 3 sides) and two squares ( 4 sides). Therefore, its name is 3.3.3.4.4 or simplified $3^{3} \cdot 4^{2}$. The list of polygons always begins with the one with least number of edges, following an order from smallest to largest. For the above example, it is uses $3^{3} \cdot 4^{2}$ instead of $4^{2} \cdot 3^{3}$.

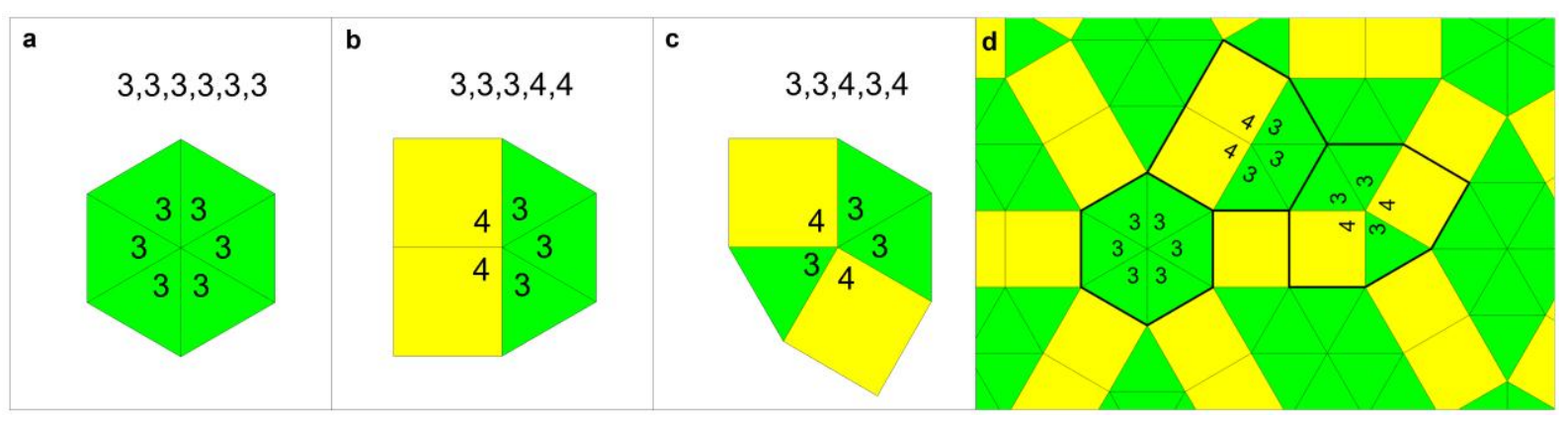

Fig. 1. Nomenclature of modules 3.3.3.3.3.3 / 3.3.3.4.4 / 3.3.4.3.4 after Cundy and Rollett and composition

In Table 1, it is presented the simplified nomenclature that currently applies to those tilings that have been incorrectly claimed as the only 14 existing demiregular tessellations (there are infinite), organized according to the numbering used primarily by Critchlow (1969). 


\section{Disadvantages of Cundy \& Rollett's nomenclature}

A major disadvantage of the conventional notation is the extensive length implied in the name of demiregular tessellations. Let's take the example of a certain mosaic (No. 12); it would be appointed as follows: 3.3.3.3.3.3 / 3.3.3.4.4 / 3.3.4.3.4 \#1. In addition to its quite long name, this denomination is not unique, and indeed the particle \#1 serves to differentiate it from tiling No. 11 , which responds to the same classification but with particle \#2.

Another complication involved in Cundy and Rollett's designation is that the generation of mosaics from the two or three basic tessellations (defined around each vertex) is not at all intuitive or easy. For example, in the case of mosaic No. 12 above, from the current nomenclature we get the three seed stem shown in Fig. 1(a) to Fig. 1(c). As it can be appreciated, if we met with these three figures and we had to generate a tiling from them, apart from not providing a single and unique configuration as explained in the preceding paragraph, the solution would not be at all intuitive or easy. Not only the connection of one module to another is complicated, but even the orientation of the modules may vary significantly.

As can be seen in Fig. 1(d), the composition of the mosaic in question is not immediate, because the orientation and location of these units is quite arbitrary.

\section{Proposal for a new nomenclature of tessellations}

Below, a new proposal is presented to name, define and generate systematically and unequivocally demiregular mosaics. In such a way, two different purposes will be achieved: on the one hand, shorten the names defining such tiles; and on the other hand, this notation is sufficient to allow the generation of specifically and unmistakably geometric configuration of every one of the tessellations. This proposal, consistent and coherent with the current notation used for the Archimedean mosaics, is based on the use of symmetries and translations (all with replica) that applied to a minimum number of suitably chosen polygons generate the overall result, which can be extended as much as desirable by repeating these geometric operations. 


\section{Practical methodology from a graphical example}

To explain the methodology in a simple manner, it shall be used an example of a specific 3-uniform mosaic, in this case the tile No. 12 mentioned above, which responds to the following headings: $3^{6} /$ $3^{3} \cdot 4^{2} / 3^{2} \cdot 4.3 .4$ \#1after Cundy and Rollett or $3-4-3,4-3^{2} / 30 / 60$ after the proposed nomenclature.

\section{Stage 1: Location of seed polygon (e.g. 3-...)}

To begin the procedure, the main vertical axis is set up (along the "y" axis usually considered in the Cartesian coordinate system). At the origin, the seed polygon (usually the one with larger number of edges) is placed and is the first digit specified in the proposed notation (in our example, a triangle, so number 3). At the time of placing the first polygon, it will be done in such a way that the two sides that intersect the horizontal axis "x", stay perpendicular to that axis (see Fig. 2). In the case of the triangle, having an odd number of edges, the left-hand edge will be the one perpendicular to the $x$ axis and will be aligned with the vertical axis " $y$ ".

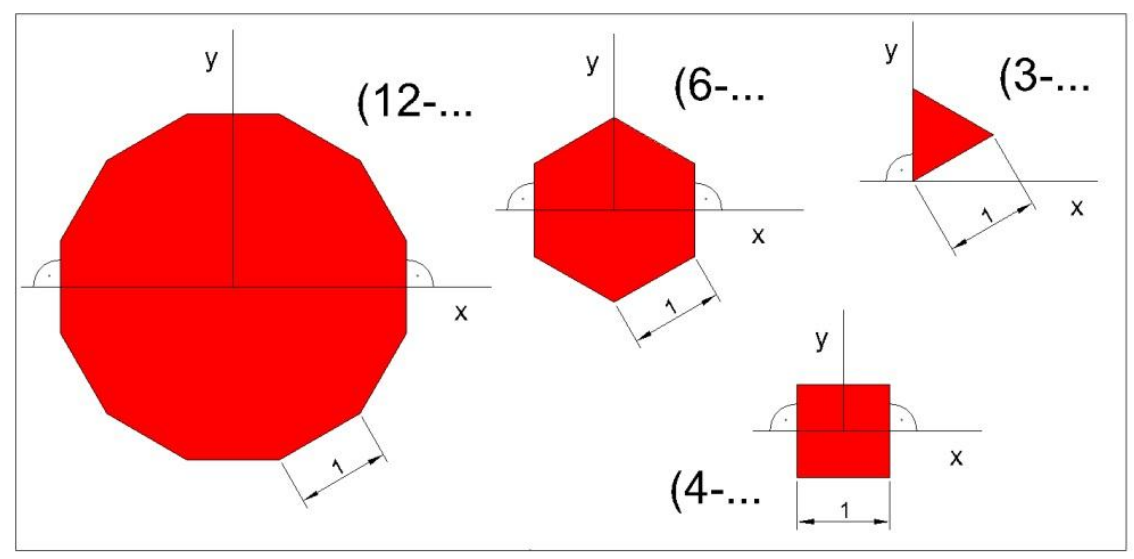

Fig. 2. Initial disposition of seed polygons

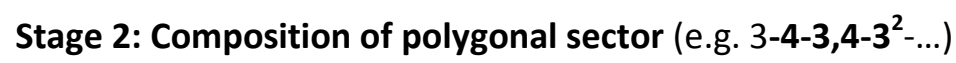

In this stage, we distinguish several phases: in each one of them, a series of polygons will be added to the others, being that phase separation represented by a dash (-). Just as in the conventional nomenclature, the repetition of adjacent polygons is denoted by a superscript. Once the seed polygon is located at the origin (Fig. 3(a)), which we considered as the first step (i) in the next phase (ii) the next set of polygons is attached to the edges of the seed polygon, starting from the vertical 
axis "y" and going on clockwise. In our example, a square (4) would be attached to the first edge of the first polygon (3) (Fig. 3(b)). In the third step (iii) next polygons are added to the free sides of the polygons of the previous phase (ii), always in the clockwise direction and into the upper right quadrant; in this case, a triangle and a square (3.4) will be built around the square. The third free edge of the square would remain empty, because there are not further specified polygons to add (Fig. 3(c)). Finally, the fourth phase (iv), add the last two equilateral triangles $\left(3^{2}\right)$ around the two polygons introduced in the previous step (iii) as shown in Fig. 3(b). When any of the intermediate edges of a polygon must remain free, it will be designated with a zero to preserve their exempt status, as in the mosaic No. 1, called 12-0.3/45/90

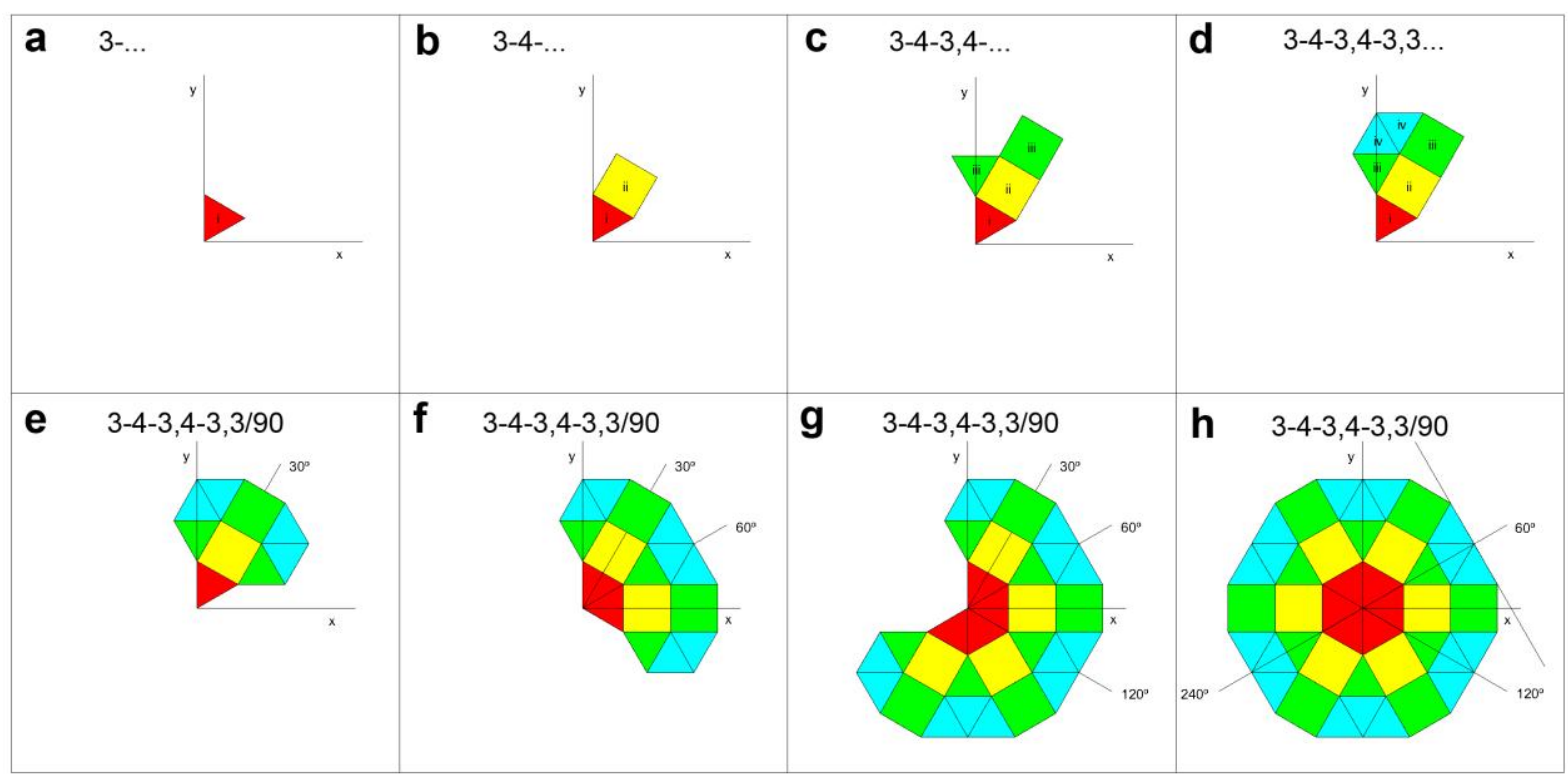

Fig. 3. Generation in phases of the basic tiling module of mosaic No. 12: $3-4-3,4-32 / 30 / 60$

Stage 3: Symmetries of polygonal sector (e.g. 3-4-3,4-32/30...)

Having obtained the basic polygonal sector, we proceed to apply the symmetries (characterized by the slash (/) followed by the respective angle of symmetry in sexagesimal degrees), in this case, a reflection on an axis from the origin at $30^{\circ}$ with the vertical (Fig. 3(e)). This symmetry is repeated continuously about axes that form angles of inclination duplicating the inclination of the previous one, i.e. $60^{\circ}, 120^{\circ}, 240^{\circ}$, etc. (Fig. $3(\mathrm{e})$ to $(\mathrm{g})$ ) to complete the 360 -degree mosaic of the basic module (Fig. 3(h)). 
As one can realize from Fig. 4 , in the case of mosaic No. 3, 12- $3^{2}, 4-4,3,0,3 / \mathrm{r} 90 / \mathrm{t} 90$, the composition of the basic tiling cell is not achieved by symmetries, but by rotations of $90^{\circ}$ of the initial polygonal sector. This exception is represented in the notation by including an $r$ (rotation) before that angle, staying as $/ \mathrm{r} 90$.

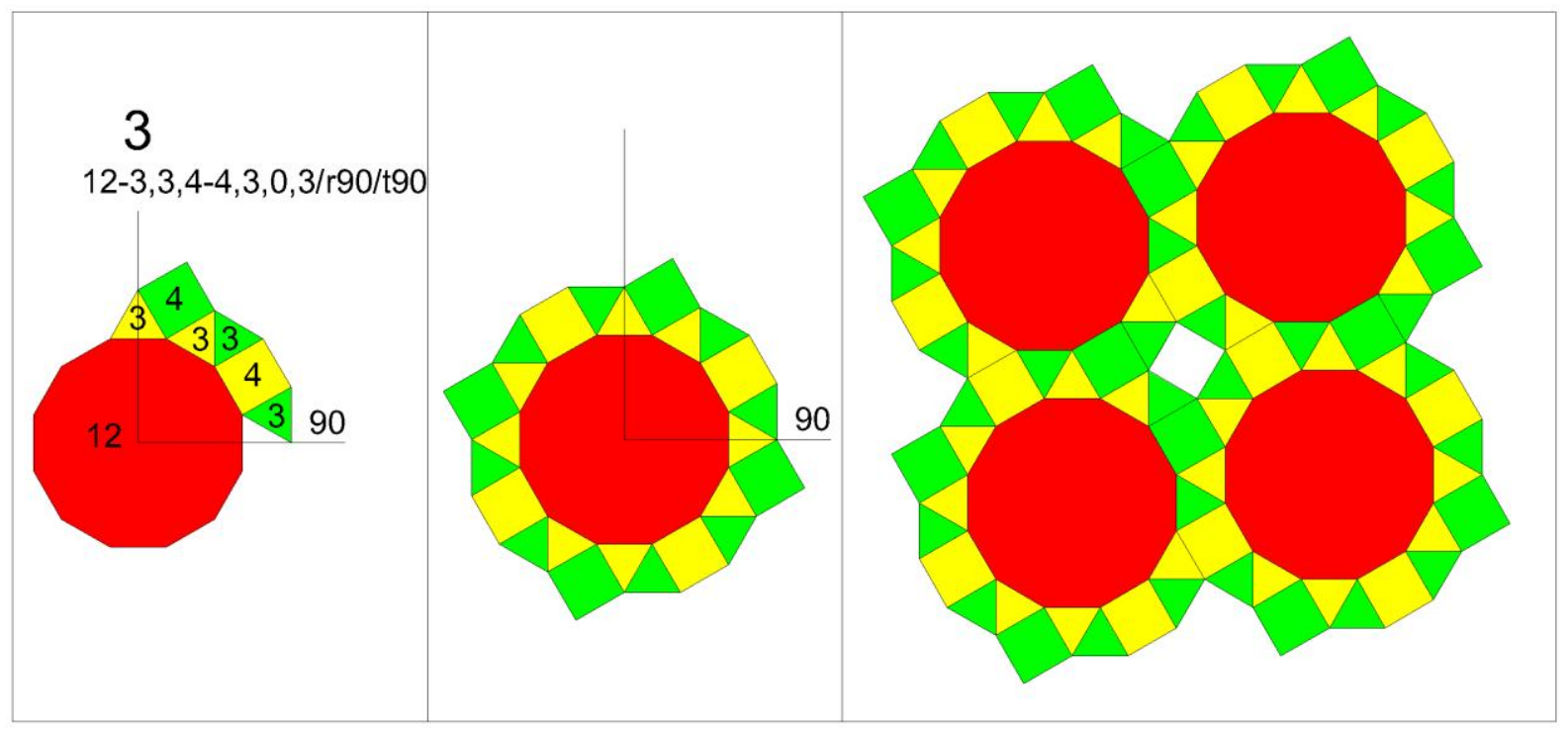

Fig. 4. Generation of mosaic No. 3

Stage 4: Symmetries of the basic tiling module (e.g. $\left.3-4-3,4-3^{2} / 30 / 60\right)$

Finally, we proceed to make the last sequence of symmetries of the basic tiling modules or cells in order to obtain the whole tessellation, following another axis perpendicular to the one forming certain number of degrees with the vertical ( $y$ axis), in our case on an axis that is perpendicular to the one forming $60^{\circ}$ with the vertical axis and passing through the origin. This axis used for the reflection of that cell, is located on the edge of the basic tiling module so that it does not create new polygons overlapping between the generated and original cells.

Once this symmetry is performed, the process will be repeated similarly with respect to other axes that are perpendicular to the multiples of the original, so as to cover the entire plane without overlaps or gaps (not possible to be filled with basic polygons).

In our example, it is illustrated how the starting position of the basis tiling module of Fig. 3(h) yields the composition shown in Fig. $5(\mathrm{a})$ by a reflection about an axis that is perpendicular to line at $60^{\circ}$ 
with the vertical and passing through the origin. From this configuration, a new symmetry about a similar axis, but at $120^{\circ}$, produces Fig. 5(b). And similarly, working with axes at 180, 240을 300 , we formed the mosaics shown respectively in Fig. 5(c) to (f).

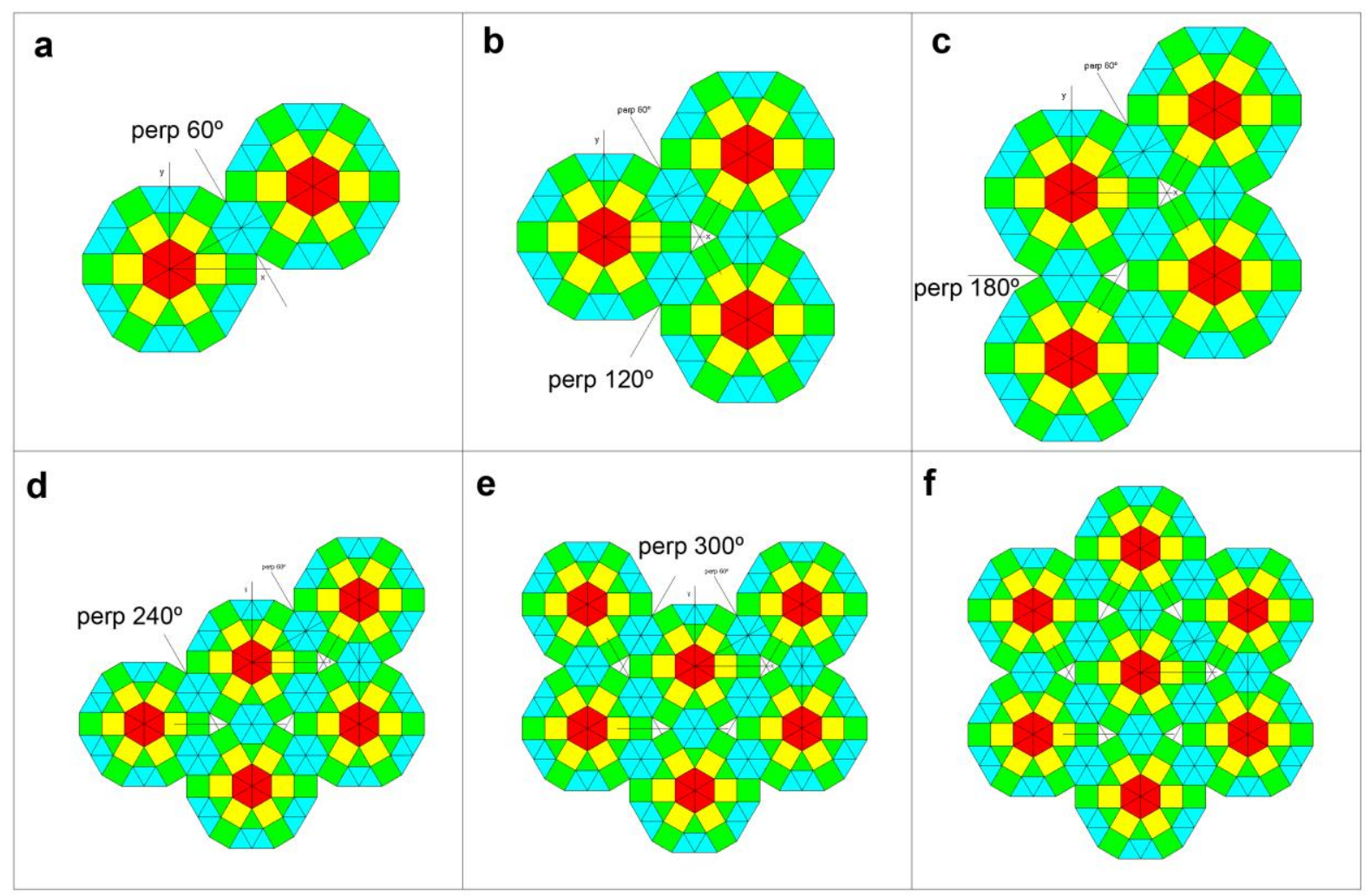

Fig. 5. Generation by symmetries, with the basic tiling module, of mosaic No. 12 : $3-4-3,4-3^{2} / 30 / 60$

It would be possible to substitute the name of these reflection isometries by different ones, such as those raised by Joyce (1997) through the 17 plane symmetry groups, or the system adopted by the IUC (International Union of Crystallography) in 1952. However, we would risk again obtaining a not very intuitive and hardly reproducible solution.

In some of these sets of reflections, small spaces stay blank, but single polygons inserted into these gaps are automatically identified by the actual shape of the contour. For example, in Fig. $5($ b), there is an empty small portion of the mosaic that is easily identified as an equilateral triangle, so it is not inconvenient at all to complete the composition.

There is another exception for the same mosaic No. 3 mentioned above; in addition to being required a rotation of $90^{\circ}$ to obtain the basic module, generation of the full tessellation is not made 
by symmetries, but by translations. This variant will be represented by the inclusion of a $t$ (translation) before the angle considered, leaving the notation /t90. In conclusion, this special mosaic would be named as $12-3,3,4-4,3,0,3 / \mathrm{r} 90 / \mathrm{t} 90$.

\section{New denomination of demiregular mosaics}

Applying the rules referred to in paragraph above, we obtain a new notation for demiregular mosaics, which will be detailed in Table 1 in comparison with current standard nomenclature and following the ordinal numbers originally used by Critchlow (1969):

\begin{tabular}{|c|c|c|c|}
\hline \multirow{2}{*}{$\begin{array}{l}\text { No. } \\
\text { Mos. }\end{array}$} & Cundy \& Rollet's nomenclature & \multirow{2}{*}{$\begin{array}{l}\text { No. } \\
\text { Mos. }\end{array}$} & Cundy \& Rollet's nomenclature \\
\hline & New proposal for nomenclature & & New proposal for nomenclature \\
\hline \multirow{2}{*}{1} & $3.12 .12 / 3.4 .3 .12$ & \multirow{2}{*}{8} & 3.3.3.4.4 / 3.3.4.3.4 / 3.4.6.4 \\
\hline & $12-0,3 / 45 / 90$ & & $6-4,4-3,3,3,3-0,0,4,3,4-0,3,3,6-0,4 / 90 / 90$ \\
\hline \multirow{2}{*}{2} & 3.3.3.3.3.3 / 3.3.4.12 & \multirow{2}{*}{9} & 3.3.3.3.3.3 / 3.3.4.3.4 \#2 \\
\hline & $12-3,4-3 / 30 / 30$ & & $3-4,3-3,3,3,4 / 90 / 90$ \\
\hline \multirow{2}{*}{3} & 3.3.4.3.4 / 3.3.4.12 / 3.4.3.12 & \multirow{2}{*}{10} & 3.3.3.3.3.3 / 3.3.4.3.4 \#1 \\
\hline & $12-3,3,4-4,3,0,3 / \mathrm{r} 90 / \mathrm{t} 90$ & & $3-4-3 / 30 / 30$ \\
\hline \multirow{2}{*}{4} & 3.4.6.4 / 4.6.12 & \multirow{2}{*}{11} & 3.3.3.3.3.3 / 3.3.3.4.4 / 3.3.4.3.4 \#2 \\
\hline & $12-6,4 / 30 / 60$ & & $4-3,3-3,4-4,3,3-0,3,4,3 / 90 / 90$ \\
\hline \multirow{2}{*}{5} & 3.3.3.3.3.3 / 3.3.4.12 / 3.3.4.3.4 & \multirow{2}{*}{12} & 3.3.3.3.3.3 / 3.3.3.4.4 / 3.3.4.3.4 \#1 \\
\hline & $12-3,4,3,4-3,3,3,3 / 90 / 90$ & & $3-4-3,4-3,3,3 / 30 / 60$ \\
\hline \multirow{2}{*}{6} & 3.3.6.6 / 3.6.3.6 & \multirow{2}{*}{13} & 3.4.6.4 / 3.4.4.6 \\
\hline & $6-3-0,6 / 90 / 90$ & & $6-4-3,4-6 / 30 / 60$ \\
\hline \multirow{2}{*}{7} & 3.3.4.3.4 / 3.4.6.4 & \multirow{2}{*}{14} & 3.3.3.4.4 / 3.4.6.4 \\
\hline & $6-4-3,3 / 30 / 60$ & & $6-4-3-3 / 30 / 30$ \\
\hline
\end{tabular}

Table 1. Comparison between Cundy \& Rollet's nomenclature and new proposal

\section{Congruency with Archimedean tessellations}

As pointed out in previous sections, the nomenclature proposed here is applicable not only for demiregular tessellations, but it is also compatible with the Archimedean, i.e. the regular and semiregular (uniform) tilings. Peculiarities lie in considering that for the latter, there is not a seed polygon tile, so there is no need of a dash (-) to separate phases; consequently, there is only one step to achieve, and polygons are cyclically listed, creating just a single type of vertex. Thus, it is possible to generate automatically, without additional symmetries, the so-called basic tiling module of the nuniform case; from this stage, repetition in the plane is obvious and intuitive, so the second set of symmetries is also omitted. 
In summary, the notation of the 11 Archimedean tiles would remain unchanged from that of Cundy and Rollet, being sorted by the number of polygons at each vertex occurring as follows: $3,12^{2}$; $4,6,12 ; 4,8^{2} ; 6^{3} ;(3,6)^{2} ; 3,4,6,4 ; 4^{4} ; 3^{4}, 6 ; 3^{3}, 4^{2} ; 3^{2}, 4,3,4 ; 3^{6}$

\section{Duals: Laves / Catalan mosaics}

A dual of a given tiling is formed by joining the centers of the polygons that compose it. Although duality is not the main subject of this work, we propose a denomination as a continuation of the nomenclature exposed above. As mentioned above, Cundy and Rollett suggested adding a prefix (V.) to the notation of a polyhedron or a mosaic to name their corresponding dual. However, we prefer to adopt the nomenclature proposed by Grünbaum and Shephard (1986), using brackets to distinguish a particular tessellation from its dual. For example, [3-4-3,4-3,3/30/60] will describe the corresponding dual of the mosaic 3-4-3,4-3,3/30/60, and so generally.

\section{Generation of DLG after Otero}

According to Otero et al. (1992), approaches to generate new double layer grids are not exactly very straightforward, and can be really difficult and challenging depending on the ability of the designer, his practice and luck.

The very first important data to know is the number of degrees of freedom disposed to carry out that task. Otero argues that with just three set of information, it is possible to define the generation of any DLG, which are:

- the mosaic of diagonal bars

- the rule of alternations to define which vertices are in which layer

- the law determining the way for joining the vertices on each chord.

Otero's contribution was to find a new approach to the generation of flat mesh, reducing to one dimension the until then tough three-dimensional formulation norm of filling the space with polyhedral cells. By doing so, a fertile panorama was accessible for designing new DLG, leaving open a broad range of possibilities for defining the laws and rules that he just started to point out. 
In which follows, a revision of Otero's work will be accomplished, complemented by a new approach made by the author and a new and systematic nomenclature to define the generation of DLG in a synthesized and unique manner. This methodology will permit to create and entitle without uncertainty new configurations of DLG.

\section{Mosaic of diagonal elements}

For the election of the mosaic of diagonal elements, there will not be a limitation to regular tessellations, as usually. On the contrary, semiregular and demiregular patterns will be utilized, as well as their duals: equifacial and semiequifacial respectively. The nomenclature employed will be the one suggested in previous paragraphs.

While the previous notation responds to a topological point of view (vicinity), the following variants that we are now considering will contemplate a geometrical perspective (taking into account angles and dimensions):

$(m, n, p, \ldots)^{d 1} \quad$ mosaic $(m, n, p, \ldots)$ rotated d1 degrees, like in the grid Space Deck, which would be $\left(4^{4}\right)^{45}$.

$(m, n, p, \ldots: a / b / c / \ldots)$ mosaic $(m, n, p, \ldots)$ with irregular sides whose lengths follow the proportion a/b/c/... An example for using this nomenclature would be the grid Mero I of Fig. 12 (e), with a diagonal mosaic made of rectangles, always from a top view, with sides in proportion 1 to root square of two, rotated 45‥ Its symbolic nomenclature would be $\left(4^{4}: 1 / \sqrt{ } 2\right)^{45}$.

\section{Law for locating the vertices}

Once defined the first element of the tern, the tessellation of diagonal bars, the laws concerning the disposition of vertices are the following (it is provided Otero's nomenclature (1990) and the abbreviator that will be used from this point):

b.1) All the vertices of the mosaic belong to both chords, top and bottom. Apart from trivial, this choice converts the new DLG in a truss-like structure, not spatial. 
b.2) Vertices of each polygon of the tessellation are located alternately in the top or bottom string of the grid. This pattern can't be followed when any of those polygons has an odd number of sides.

b.3) All the vertices of the mosaic belong to one of the chords, (e.g. lower) while on the other layer (e.g. upper) are located alternately. Vertical elements could be suppressed.

b.4.) All the vertices of the mosaic belong to one of the chords, (e.g. lower), and the middle points between them belong to the other layer (e.g. upper). This option can be especially used in cases where the option $\mathbf{b . 2}$ is not available, i.e. when there are polygons with odd number of sides.

Due to the need of structuring the different possibilities for defining the DLG after those rules, here it is proposed a new nomenclature that describes the statements made by Otero but in a synthesized and unique manner. This is very important in order to avoid ambiguity in the creation of these kinds of structures, especially concerning to the generation of new configurations of DLG.

Symbols used for defining the graphic configuration of the grids will be those shown in Fig. 6 .

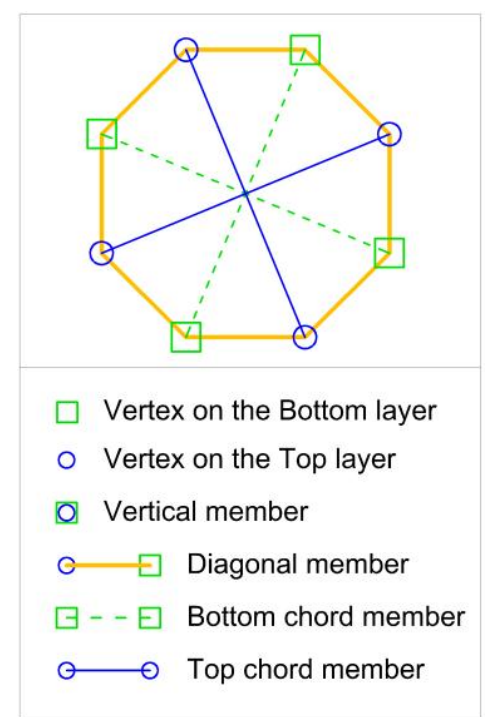

Fig. 6. Set of symbols for the elements of a DLG

Sometimes it could be useful to know on which layer of the grid is applied any of this laws (especially concerning cases b.3 or b.4); anyhow b. 3 could be considered a conjunction of cases b.1 (for just one layer) and b.2 (for the other), while in b.4 it is also included the option b.1 for one of the strings. 
Therefore, it could be convenient to apply the different rules to each one of the layer independently.

Doing so, it will be possible to formulate the precedent laws for each cord as follows:

e) Everyone: every vertex of the mosaic belongs to that particular layer.

a) Alternated: the vertices belong or not, alternately, to that particular layer. Another variant is applicable only to polygons with a number of sides ( $n$ ) multiple of the frequency (v) of the alternancy. Apart from the usual case $(v=2)$, the rest of the cases are just applicable to polygons with $n$ sides, $n=k \cdot v($ for $k=2,3,4, \ldots)$

a) $\left(=a^{2}\right)$ : usual case, frequency $\mathbf{v}=\mathbf{2}$, i.e. alternated normally (one every two). See case $(4,6,12)-$ Ba $1 a^{3}{ }_{12} 1-T \underline{a} 1 a^{4}{ }_{12} 1$ of Fig. 7.

$\left.a^{3}\right)$ frequency $v=\mathbf{3}$, i.e. one every three, etc. (see case $(4,6,12)$-Ba $1 \underline{a}^{3}{ }_{12} 1-T a 1 a^{4}{ }_{12} 1$ of Fig. 7)

$\left.a^{4}\right)$ frequency $v=4$, i.e. one every four, etc. (see case $(4,6,12)-$ Ba1 $^{3}{ }_{12} 1-T a 1 \underline{a}^{4}{ }_{12} 1$ of Fig. 7)

m) Middle: the middle points between the vertices of the mosaic belong to that particular layer.

s) Segregated: In some double layer tensegrity grids (DLTG), like the one of Fig. 8 composed by modules of half cuboctahedron (or T-4-Pyramid), the location of nodes is totally different; they are not on the vertices, and they are located every five middle positions (s5). This option produces an intertwist or interweaving of the diagonal members, crossing each other at different levels of the plane.

$(4,6,12)-\mathrm{Ba} 1 \mathrm{a}_{12}^{3} 1-\mathrm{Ta} 1 \mathrm{a}_{12}^{4} 1$

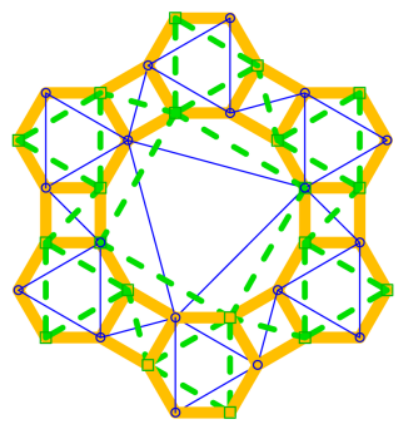

$\mathrm{Ba} 1 \mathrm{a}^{3} 1$

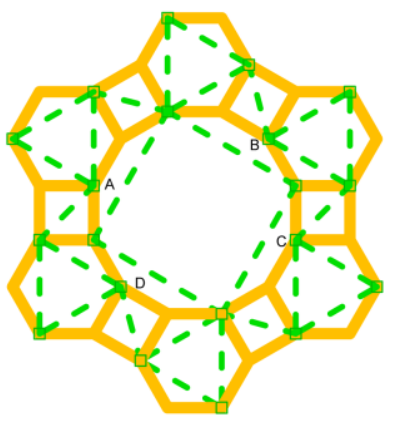

$\mathrm{Ta} \mathrm{a}_{12}^{4} 1$

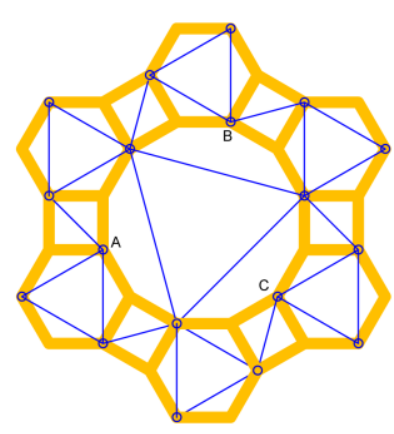

Fig. 7. Alternate law for locating the vertices with frequencies three (a3) and four (a4) 


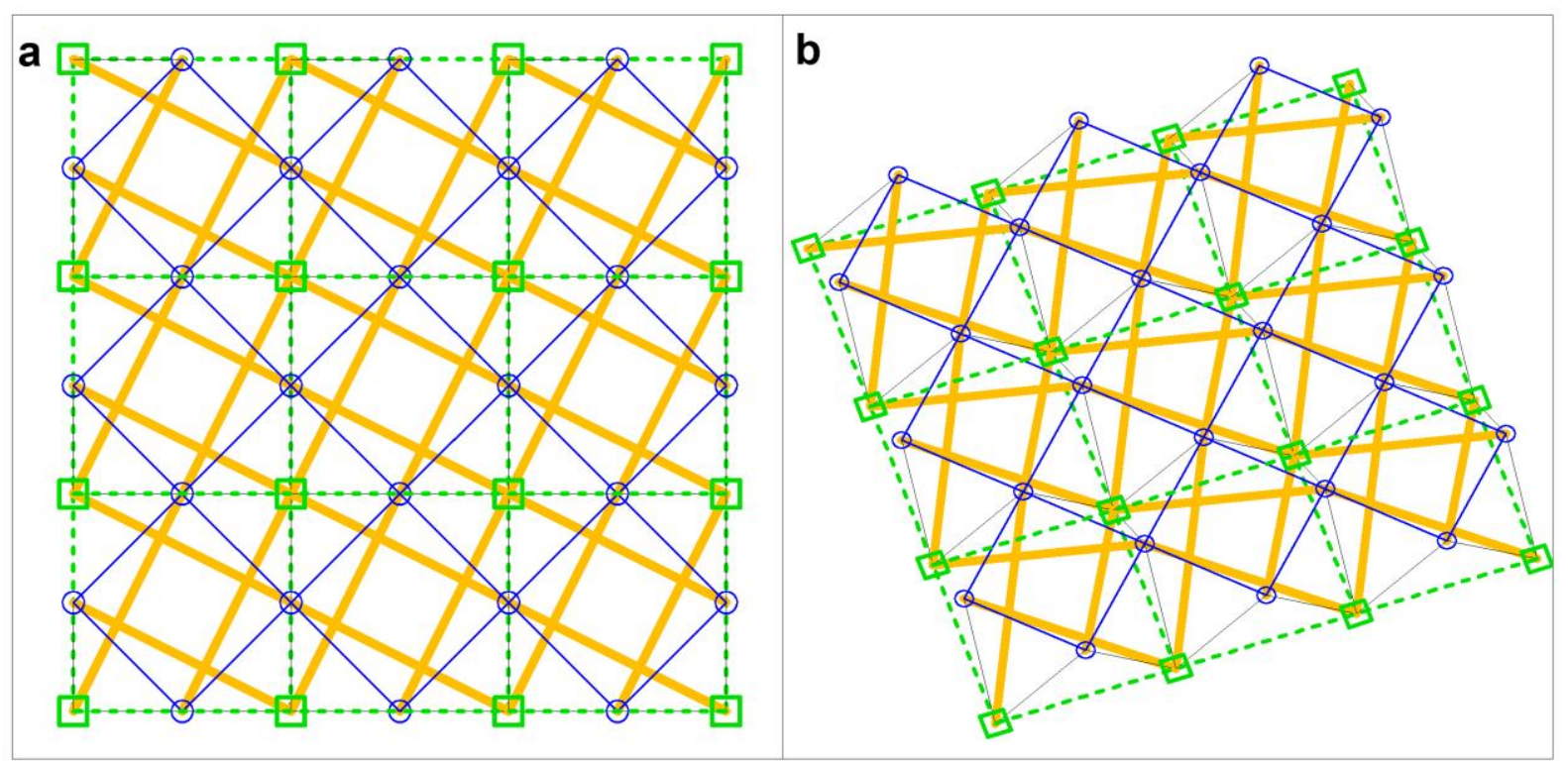

Fig. 8. DLTG made by modules of half cuboctahedron. a) Plan view. b) Perspective

According to Fig. 7, it can be observed that the location of vertices alternately, for frequencies greater than 2, can form configurations where polygons of bottom or top layers are not convex. This is due to the fact that nodes $A, B, C$ and $D$ belong to the squares and hexagons, but not to the dodecagons.

Formulated like that, Otero's proposals are observed on this nomenclature after the disposition of the upper and lower strings respectively: $b .1=e+e ; b .2=a+a ; b .3=e+a ; b .4=e+m$ It is possible that in some layers the disposition of vertices was a mix of two options, like in grid Friedman, where there is a combination of options $\mathbf{e}$ and $\mathbf{m}$, so all the vertex of the diagonal tessellation, as well as the middle points between them, belong to the top layer.

Occasionally, when polygons are non-regular, the disposition of nodes in case a (alternated order) is not arbitrary, and it is compulsory to decide the distribution of the vertices on the bottom and top layer. For these cases, like in the grid Hexagonal, it will be necessary to explicit as an exponent of the letter $a$, any of these options:

S) Alternated vertices are separated by the shortest distance

L) Alternated vertices are separated by the longest distance 


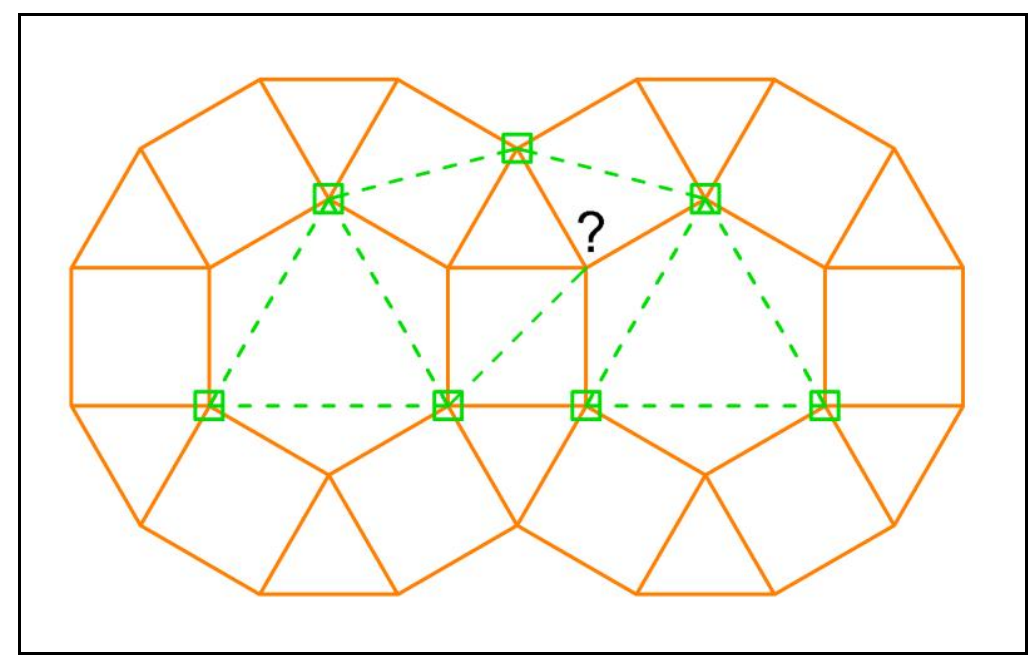

Fig. 9. Inconsistency of grids with odd number sides polygons when using law a.

The need of polygons with an even number of sides for case a (alternated) is shown, for instance, when working with mosaic $(3,4,6,4)$, where an inconsistency is produced due to the lack of symmetry of the whole set (Fig. 9).

\section{Law for joining the vertices}

The third degree of freedom is the law for joining the vertices on each layer. Otero (1990) proposed two tendencies, just to expose some of the possibilities, as his main goal was not to generate an exhaustive list of conceivable connections, having into account mainly the two or three directions defined by nearest nodes:

c.1) Connecting each vertex to its neighbours.

c.2) Establishing an alternative connection.

Sometimes, especially in non-regular tessellations, a certain direction chosen for joining two vertices has no continuity in the rest of the mosaic (Fig. 10). Thus, it is necessary to find another methodology for relating any vertex to its neighbours. 


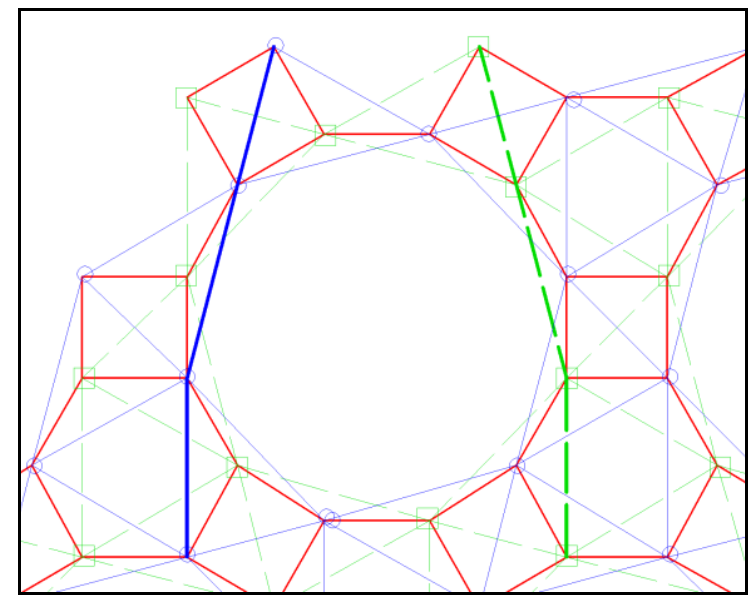

Fig. 10. Discontinuity of directions joining vertices in DLG $(4,6,12)-B a 1-T a 1$

Henceforth, it will be considered each law of connection of vertices different and independent from the bottom chord (which will be defined after the letter B) to the top chord (preceded by letter $\mathrm{T}$ ), so that the whole grid will respond to a combination of the configuration of both laws.

In order to obtain a more ample approach comprising those two options, another proposal and symbolic definition is presented in next lines.

1) Linking a vertex to the nearest one of each polygon.

2) Linking a vertex to the second nearest one of each polygon.

n) Linking a vertex to the n-order nearest one of each polygon.

If the joining rules are different within every polygon of a layer, the number of the connecting law will have a sub-index characterizing the correspondent polygon. For instance, $1_{4} 2_{12}$ means that the vertices of quadrilaterals are joined to their nearest neighbours, while the connection of vertices of dodecagons reaches the second nearest distance.

These options are not restrictive, and the connections of vertices can be a combination by overlapping of some of them. As an example, in Fig. 11 it is shown some of the basic linking configurations for the diagonal mosaic $(4,8,8)$ : a1, a2 ${ }_{8}$, e1, e2, e3, e4, m1, m2, etc. From their combinatory composition, many others can be produced by superposition: a12, a $1_{4} 2_{8}$, e12, e13, e14, e23, e24, e34, m12, m13, m23, etc. Fig. 12 illustrates some of them. 


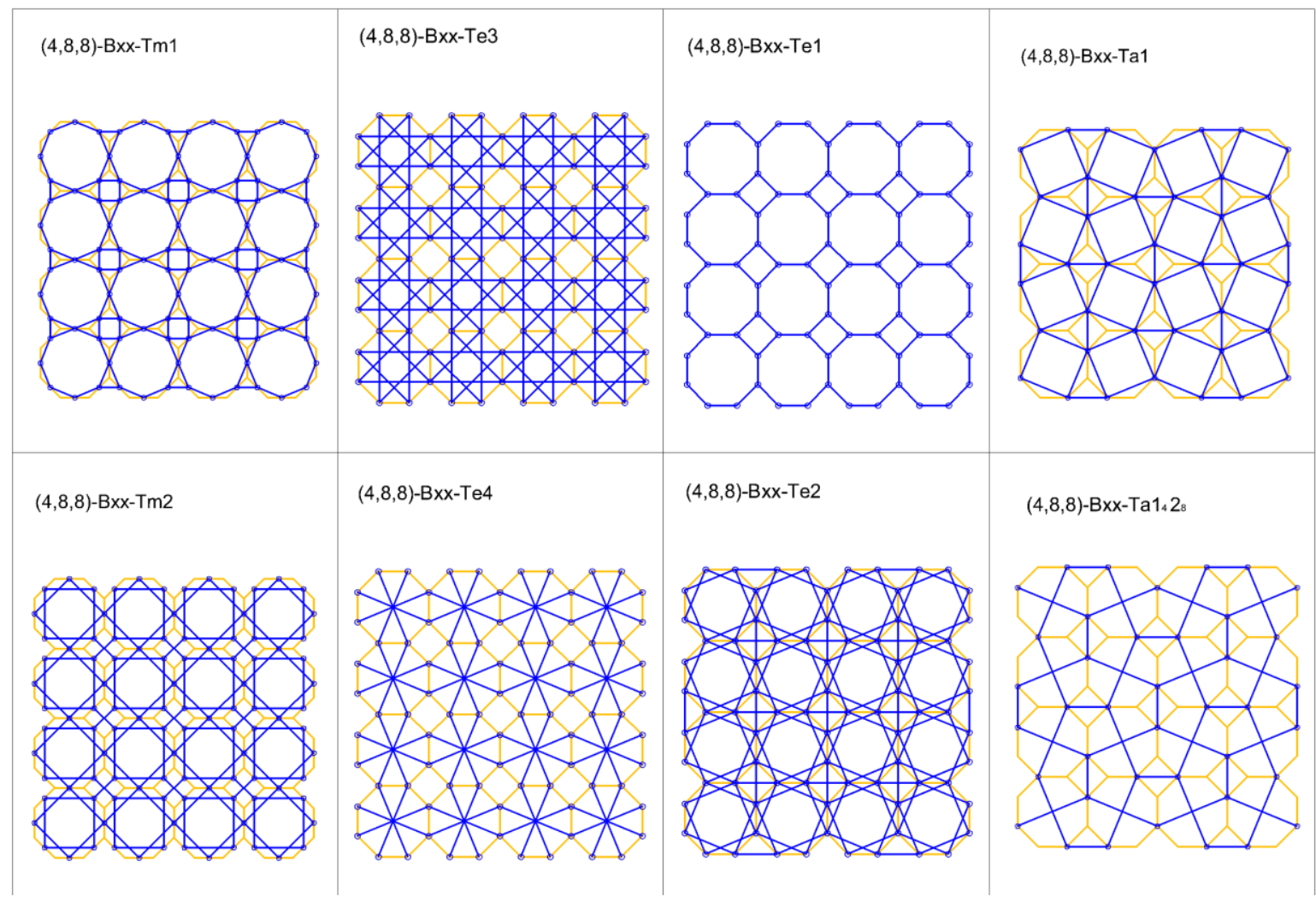

Fig. 11. Generation of bottom or top layers from the mosaic $(4,8,8)$

Moreover, comprised in any of the precedent situations, in some occasions the connection is established only in some directions, and not in others. In those cases, moving again from a topological to a geometrical point of view, the numbers will have an exponent with the direction or directions of connection (e.g. $1^{45,60}$ would express that the node is linked to the nearest vertices aligned $45^{\circ}$ and $60^{\circ}$ with itself), like in grid Wachsmann (Fig. 12(b)). When not all the vertices have the same directions of relationship, the exponent will be expressed like, for instance, $2^{a(45: 135)}$, which means that a node is linked to the second nearest vertices aligned $45^{\circ}$ and the adjacent nodes would do it with the angle 135․ For clarification, see grid Friedman (Fig. 12.(f)).

In some instances (e.g. rhombs composed by two adjacent equilateral triangles), the nearest vertices could be adjacent or opposite. To avoid this ambiguity, the choice will be expressed as an exponent with the nomenclature:

Op) Linking a vertex to the opposite one of each polygon.

Ad) Linking a vertex to the adjacent ones of each polygon. 
A very clear example is the grid Unit IV (Fig. 12(d)), in which some of the nodes of the bottom are linked to all the six nearest neighbours, while some others connects only with the three that are adjacent in the polygon in which are included.

Some other times, the connection follows a definite pattern dictated by certain directions inside the polygon. In those cases, the directions will be indicated as exponents separated by commas. If those patterns are not the same on each polygon (they are usually zigzagging), the corresponding data will be anticipated by the letter $a$ (of alternated) and separated by a dash.

For an example, even if we can agree that the grid Friedman of Fig. $12(f)$ is $\left(4^{4}\right)$-Be1-Tem12, it could also be written as: $\left(4^{4}\right)-B e 1-\operatorname{Tm} 12^{a(0,90)}$

$(4)^{4}-\quad$ Diagonal mosaic is regular, four squares (4 sides) coinciding on each vertex.

Be1 - Vertices of Bottom (B) layer on every (e) vertex of the diagonal mosaic. Each one of them is joined to its nearest (1) nodes.

$\operatorname{Tm} 12^{\mathrm{a}(0,90)}$ - Vertices of Top $(\mathrm{T})$ layer on middle $(\mathrm{m})$ points between vertex of the diagonal mosaic. Each one of them is joined to its nearest (1) nodes and to the second (2) nearest neighbours, alternately on horizontal (0) and vertical (90).

In order to write the complete symbolic generation of a DLG, it will be presented another example of the grid Wachsmann (Fig. 12(b)): $\left(4^{4}\right)^{45}-$ Ba12 $^{45}-$ Ta12 $^{135}$

$\left(4^{4}\right)^{45}$ - Diagonal mosaic is regular, four $\left({ }^{4}\right)$ squares (4 sides) coinciding on each vertex, and rotated 45ㅇ․

Ba12 $2^{45}$ - Vertices of Bottom (B) layer on alternate (e) vertex of the diagonal mosaic. Each one of them is joined to its nearest (1) nodes and to the second nearest neighbours at 45 ․

Ta12 $2^{135}$ - Vertices of Top $(T)$ layer on alternate (a) vertex of the diagonal mosaic. Each one of them is joined to its nearest (1) nodes and to the second nearest neighbours at 135‥ 


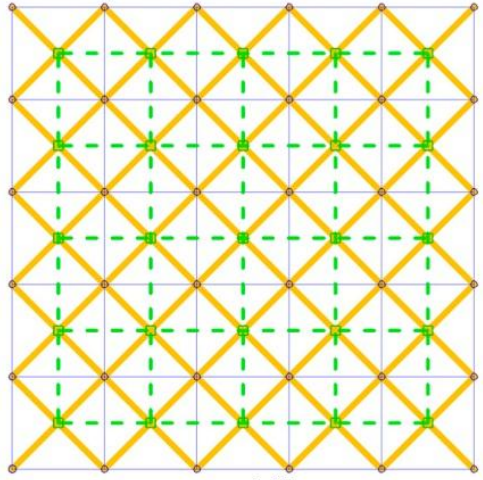

a) Space Deck: $\left(4^{4}\right)^{45}-\mathrm{Ba} 1-\mathrm{Ta} 1$

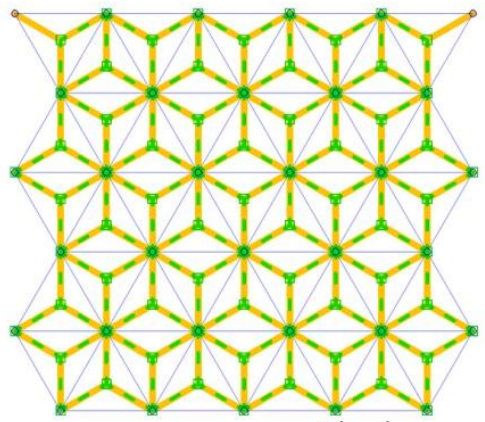

d) Unit-IV: $(3,6,3,6)-\mathrm{Be}^{\mathrm{ad}}-\mathrm{Ta}^{\mathrm{L}} 1$

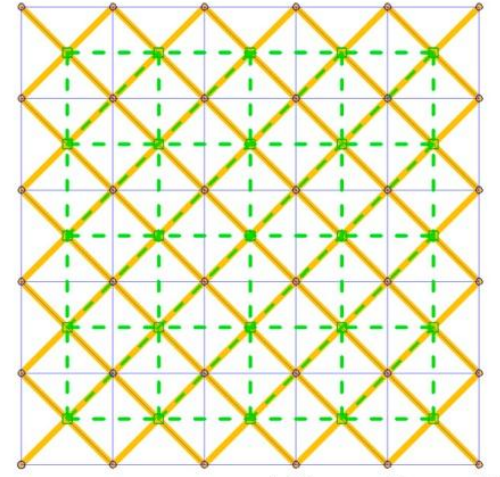

b) Wachsmann: $\left(4^{4}\right)^{45}-\mathrm{Ba} 12^{45}-\mathrm{Ta} 12^{135}$

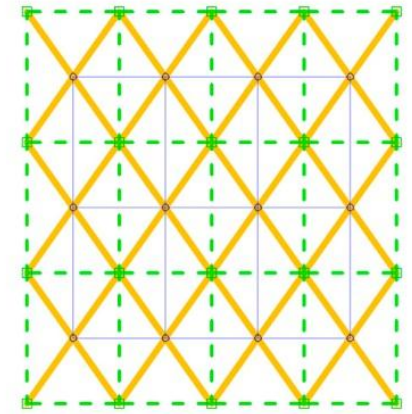

e) Mero-I: $\left(4^{4}: 1 / \sqrt{2}\right)^{45}-\mathrm{Ba} 1-\mathrm{Ta} 1$

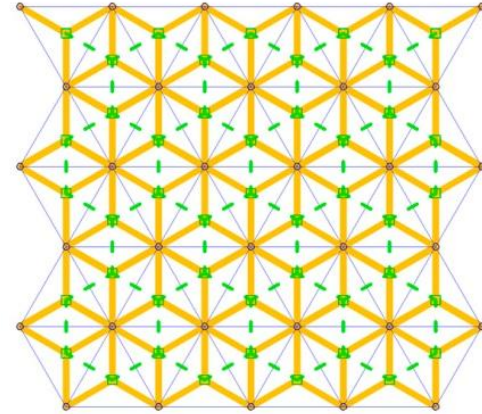

c) Hexagonal: $(3,6,3,6)-\mathrm{Ba}^{\mathrm{S}} 1-\mathrm{Ta}^{\mathrm{L}} 1$

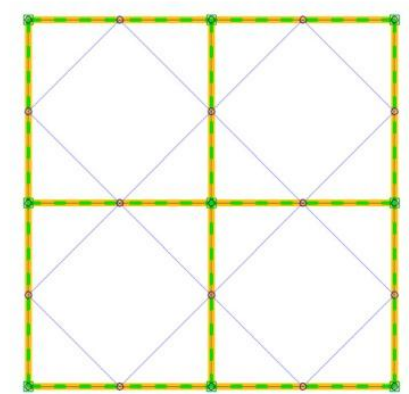

f) Friedman: $\left(4^{4}\right)^{45}-\mathrm{Be} 1-$ Tem 12

Fig. 12. Some DLGs with their current name and the proposed nomenclature

\section{Conclussions}

The main conclusion of this paper is that there is a huge potential for this new nomenclature, either for tessellations or DLGs, with the possibility of recreating the whole design or structure automatically as a result of the meaning and uniqueness of its denomination. There are two important derivations, explained in following lines.

\section{Automatic Design and Visualization}

A general procedure for the automatic design and visualization of tessellations and DLGs starts by the introduction of the denomination of the structure by means of a bar code, QR code, etc. or even manually. They could be read easily by any digital device (PDA, phone, computer, etc.), so to generate the whole grid just after reading these tags, which could be printed easily anywhere.

From here, two main alternatives are pointed-out: On the one hand, the most obvious, recreating the structure with CAD software by means of the implementation of programming code. And on the 
other hand, the most immediate, reconstructing the design in the own reader device, doing the interpretation of the name and generating the 3D structure as long as its correspondent visualization. Those QR codes, for instance, could host the DLG denomination (4^4)-Be1$\operatorname{Tm} 12 a^{\wedge}(0,90)$ and the url of the web page with the program for downloading the code, program or 3D generator and viewer. An example is shown in www.tensegridad.es/DLG-tessellations/DLGtessellations.html.

\section{New configurations of DLG}

Once established the adaption of this methodology to create innovative DLG, it is indispensable to apply the procedure in a systematic manner, in order to explore the broad possibilities that it has intrinsically.

For such a mission, an algorithm will be exposed in future expositions in order to obtain new designs of grids from non-regular tessellations.

\section{Acknowledgment}

Corresponding author would like to thank the Basque Delegation of the Spanish College of Civil Engineers for their support during the time while this research was carried out.

\section{References}

Critchlow, K. (1969). Order in space: a design source book. Thames and Hudson, London.

Cundy, H. M., and Rollett, A. P. (1981). Mathematical models. Tarquin, Stradbroke.

Cuoco, D., and American Society of Civil Engineers. Task Committee on Double-Layer Grids. (1997). Guidelines for the design of double-layer grids. American Society of Civil Engineers.

Eekhout, M. (1989). Architecture in space structures. 010 Publishers, Rotterdam.

Grünbaum, B., and Shephard, G. C. (1986). Tilings and patterns. WH Freeman \& Co., New York.

Joyce, D. E. (1997). "The 17 plane symmetry groups." The 17 plane symmetry groups, <http://www.clarku.edu/ djoyce/wallpaper/seventeen.html> (Dec. 23, 2010). 
Lan, T. T. (1999). "Space Frame Structures." Structural Engineering Handbook, CRC Press LLC, Boca Raton, Florida, 24.1-24.50.

Makowski, Z. S. (1968). Estructuras espaciales de acero. Gustavo Gili, Barcelona.

Makowski, Z. S. (1981). Analysis, design and construction of double-layer grids. Applied Science Publishers Ltd., London.

Malla, R. B., and Serrette, R. L. (1996). "Double-layer grids: review of static and thermal analysis methods." Journal of Structural Engineering, 122(8), 873-881.

Otero, C. (1990). “Diseño geométrico de cúpulas no esféricas aproximadas por mallas triangulares con un número mínimo de longitudes de barra." Doctoral Thesis. Universidad de Cantabria, Santander.

Otero, C., Oti, J., Villar, F., and Otero, F. (1992). "Classical Geometry in flat and simple curved meshes." Bulletin of the International Association of Shell and Spatial Structures, 33(108), 3-31. 


\section{List of Figures and Tables}

\section{Figures}

Fig. 1. Nomenclature of modules 3.3.3.3.3.3 / 3.3.3.4.4 / 3.3.4.3.4 after Cundy and Rollett and composition

Fig. 2. Initial disposition of seed polygons

Fig. 3. Generation in phases of the basic tiling module of mosaic No. $12: 3-4-3,4-3^{2} / 30 / 60$

Fig. 4. Generation of mosaic No. 3

Fig. 5. Generation by symmetries, with the basic tiling module, of mosaic No. $12: 3-4-3,4-3^{2} / 30 / 60$

Fig. 6. Set of symbols for the elements of a DLG

Fig. 7. Alternate law for locating the vertices with frequencies three (a3) and four (a4)

Fig. 8. DLTG made by modules of half cuboctahedron. a) Plan view. b) Perspective

Fig. 9. Inconsistency of grids with odd number sides polygons when using law a.

Fig. 10. Discontinuity of directions joining vertices in DLG $(4,6,12)-B a 1-T a 1$

Fig. 11. Generation of bottom or top layers from the mosaic $(4,8,8)$

Fig. 12. Some DLGs with their current name and the proposed nomenclature

\section{Tables}

Table 1. Comparison between Cundy \& Rollet's nomenclature and new proposal 